\title{
DIFFERENTIABILITY OF SOLUTIONS OF SYSTEMS WITH IMPULSE EFFECT WITH RESPECT TO INITIAL DATA AND PARAMETER
}

\author{
by P. S. SIMEONOV and D. D. BAINOV
}

(Received 17th July 1986)

\section{Introduction}

It is characteristic for the development of many processes that in certain moments they change their state in jumps. Systems with impulse effect provide an adequate mathematical model of such processes. The investigation of these systems begins with the paper of Milman and Myshkis [7] and afterwards the number of publications dedicated to this problem rapidly increases.

In part of the papers on systems with impulses the apparatus of the generalized functions is used $[9-11,20]$.

In the papers $[2,5,8,12-19]$ the apparatus of the classical theory of the ordinary differential equations is used. In them systems with impulse effect of the form

$$
\begin{gathered}
\frac{d x}{d t}=f(t, x), t \neq \tau_{k}(x), \\
\left.\Delta x\right|_{t=\tau_{k}(x)}=I_{k}(x)
\end{gathered}
$$

are investigated where $t \in I=[0, \infty) ; x \in \Omega \subset \mathbb{R}^{n} ; \tau_{k}: \Omega \rightarrow \mathbb{R} ; f: I \times \Omega \rightarrow \mathbb{R}^{n} ; I_{k}: \Omega \rightarrow \mathbb{R}^{n}$.

The development of the qualitative theory of systems with impulse effect requires the use of theorems from the fundamental theory of these systems.

We shall note that in [8] results related to the existence, uniqueness and continuability of the soluions of system (1) are given.

In the present paper a system with impulse effect in fixed moments of time of the form

$$
\begin{gathered}
\frac{d x}{d t}=f(t, x, \lambda), \quad t \neq \tau_{k}, \\
\left.\Delta x\right|_{t=\tau_{k}}=I_{k}(x, \lambda), \quad k=1,2, \ldots
\end{gathered}
$$

is considered where $t \in I ; x \in \Omega \subset \mathbb{R}^{n}$; the parameter $\lambda \in \Lambda \subset \mathbb{R}^{m} ; \Omega$ and $\Lambda$ are domains; $0=\tau_{0}<\tau_{1}<\cdots<\tau_{k}<\cdots ; \lim _{k \rightarrow \infty} \tau_{k}=\infty$. 
A theorem of differentiability of the solutions of system (2) with respect to initial data and parameter is proved. The respective variational equations, the formula of variation of parameters and an analogue of Alekseev's formula are obtained.

\section{Preliminary notes}

We recall that the systems with impulse effect of the form (2) are characterized as follows:

1. For $t \neq \tau_{k}$ the mapping point $(t, x(t))$ moves along an integral curve of the system $d x / d t=f(t, x, \lambda)$.

2. In the moment $t=\tau_{k}$ the system is subject to an impulse effect and the mapping point instantly "jumps" from the position $\left(\tau_{k}, x\left(\tau_{k}\right)\right)$ into the position $\left(\tau_{k}, x\left(\tau_{k}\right)+I_{k}\left(x\left(\tau_{k}\right), \lambda\right)\right)$. After that, for $\tau_{k}<t \leqq \tau_{k+1}$ the solution $x(t)$ of system (2) coincides with the solution $y(t)$ of the initial value problem

$$
\frac{d y}{d t}=f(t, y, \lambda), y\left(\tau_{k}\right)=x\left(\tau_{k}\right)+I_{k}\left(x\left(\tau_{k}\right), \lambda\right) .
$$

In the moment $t=\tau_{k+1}$ the system is subject to a new impulse effect, etc.

3. In the moments $\tau_{k}$ of impulse effect, the solution $x(t)$ of system (2) is left continuous, i.e.

$$
x\left(\tau_{k}-0\right)=x\left(\tau_{k}\right), x\left(\tau_{k}+0\right)=x\left(\tau_{k}\right)+\Delta x\left(\tau_{k}\right)=x\left(\tau_{k}\right)+I_{k}\left(x\left(\tau_{k}\right), \lambda\right) .
$$

We shall use the following notation:

$x\left(t ; t_{0}, x_{0}, \lambda\right)$ is the solution of system (2) for which $x\left(t_{0}+0 ; t_{0}, x_{0}, \lambda\right)=x_{0} ; J^{+}=$ $J^{+}\left(t_{0}, x_{0}, \lambda\right)\left(J^{-}=J^{-}\left(t_{0}, x_{0}, \lambda\right)\right)$ is the maximal interval of the form $\left(t_{0}, \omega\right)\left(\left(\alpha, t_{0}\right]\right)$ where the solution $x\left(t ; t_{0}, x_{0}, \lambda\right)$ is right (left) continuable, $J\left(t_{0}, x_{0}, \lambda\right)=J^{+} \cup J^{-}=(\alpha, \omega) ; \partial f / \partial x=$ $(\partial f i / \partial x j)$ is the Jacobi matrix of the function $f(x) ; E_{n}$ is the unit $(n \times n)$-matrix; $0_{n m}$ is the zero $(n \times m)$-matrix; $\operatorname{Tr} A=\sum_{i=1}^{n} a_{i i}$ is the trace of the matrix $A=\left(a_{i j}\right)_{1}^{n} ; i\langle\alpha, \beta\rangle$ is the number of the moments of impulse effect $\tau_{k}$ lying in the interval $\langle\alpha, \beta\rangle ;|x|$ is the norm of the vector $x \in \mathbb{R}^{n}$.

\section{Main results}

\subsection{Continuous dependence of the solutions on initial data and a parameter}

Theorem 1. Let the following conditions be fulfilled: (i) The function $f: I \times \Omega \times \Lambda \rightarrow \mathbb{R}^{n}$ is continuous in $\left(\tau_{k-1}, \tau_{k}\right] \times \Omega \times \Lambda, k=1,2, \ldots$ and for every $k=1,2, \ldots$ and $\left(x_{0}, \lambda_{0}\right) \in \Omega \times \Lambda$ there exists a finite limit of $f(t, x, \lambda)$ as $(t, x, \lambda) \rightarrow\left(\tau_{k}, x_{0}, \lambda_{0}\right), t>\tau_{k}$.

(ii) The function $f$ is locally Lipschitz continuous on $(x, \lambda)$ in the domain $I \times \Omega \times \Lambda$.

(iii) For $k=1,2, \ldots$ the mapping $\Psi_{k}: \Omega \times \Lambda \rightarrow \Omega \times \Lambda,(x, \lambda) \rightarrow(z, \lambda), z=\psi_{k}(x, \lambda)=x+I_{k}(x, \lambda)$ is a homeomorphism.

(iv) For $\lambda=\lambda^{*} \in \Lambda$ system (2) has a solution $\phi(t)$ defined in $[\alpha, \beta]\left(\alpha, \beta \neq \tau_{k}, k=1,2, \ldots\right)$. 
Then there exists a number $\xi>0$ and a set

$$
U=\left\{(t, x, \lambda) \in I \times \Omega \times \Lambda: \alpha \leqq t \leqq \beta,|x-\phi(t+0)|<\xi,\left|\lambda-\lambda^{*}\right|<\xi\right\}
$$

such that:

1. For each $\left(t_{0}, x_{0}, \lambda_{0}\right) \in U$ there exists a unique solution $x\left(t ; t_{0}, x_{0}, \lambda_{0}\right)$ of system (2) which is defined in $[\alpha, \beta]$.

2. The function $x\left(t ; t_{0}, x_{0}, \lambda_{0}\right)$ is continuous for $t \in[\alpha, \beta],\left(t_{0}, x_{0}, \lambda_{0}\right) \in U ; t \neq \tau_{i}, t_{0} \neq \tau_{k}$; $i, k=1,2, \ldots$.

3. For $k=1,2, \ldots ;\left(x_{0}, \lambda_{0}\right) \in \Omega \times \Lambda ; t, \tau_{i} \in J\left(\tau_{k}, x_{0}, \lambda\right), t \neq \tau_{j}$ the following relations hold:

$$
\begin{gathered}
x(s ; \tau, y, \mu) \rightarrow x\left(t ; \tau_{k}, x_{0}, \lambda_{0}\right) \text { as }(s, \tau, y, \mu) \rightarrow\left(t, \tau_{k}+0, x_{0}, \lambda_{0}\right) \\
x(s ; \tau, y, \mu) \rightarrow x\left(t ; \tau_{k}, x_{0}+I_{k}\left(x_{0}, \lambda_{0}\right), \lambda_{0}\right) \text { as }(s, \tau, y, \mu) \rightarrow\left(t, \tau_{k}-0, x_{0}, \lambda_{0}\right) \\
x(s ; \tau, y, \mu) \rightarrow x\left(\tau_{k} ; t, x_{0}, \lambda_{0}\right)+I_{k}\left(x\left(\tau_{k} ; t, x_{0}, \lambda_{0}\right), \lambda_{0}\right) a s(s, \tau, y, \mu) \rightarrow\left(\tau_{k}+0, t, x_{0}, \lambda_{0}\right) \\
x(s ; \tau, y, \mu) \rightarrow x\left(\tau_{k} ; t, x_{0}, \lambda_{0}\right) \text { as }(s, \tau, y, \mu) \rightarrow\left(\tau_{k}-0, t, x_{0}, \lambda_{0}\right) \\
x(s ; \tau, y, \mu) \rightarrow x\left(\tau_{i} ; \tau_{k}, x_{0}, \lambda_{0}\right) \text { as }(s, \tau, y, \mu) \rightarrow\left(\tau_{i}-0, \tau_{k}+0, x_{0}, \lambda_{0}\right) \\
x(s ; \tau, y, \mu) \rightarrow x\left(\tau_{i} ; \tau_{k}, x_{0}+I_{k}\left(x_{0}, \lambda_{0}\right), \lambda_{0}\right) \text { as }(s, \tau, y, \mu) \rightarrow\left(\tau_{i}-0, \tau_{k}-0, x_{0}, \lambda_{0}\right) \\
x(s ; \tau, y, \mu) \rightarrow x\left(\tau_{i} ; \tau_{k}, x_{0}, \lambda_{0}\right)+I_{i}\left(x\left(\tau_{i} ; \tau_{k}, x_{0}, \lambda_{0}\right), \lambda_{0}\right) a s(s, \tau, y, \mu) \rightarrow\left(\tau_{i}+0, \tau_{k}+0, x_{0}, \lambda_{0}\right) \\
x(s ; \tau, y, \mu) \rightarrow x\left(\tau_{i} ; \tau_{k}, x_{0}+I_{k}\left(x_{0}, \lambda_{0}\right), \lambda_{0}\right) \\
+I_{i}\left(x\left(\tau_{i} ; \tau_{k}, x_{0}+I_{k}\left(x_{0}, \lambda_{0}\right), \lambda_{0}\right), \lambda_{0}\right) \\
a s(s, \tau, y, \mu) \rightarrow\left(\tau_{i}+0, \tau_{k}-0, x_{0}, \lambda_{0}\right)
\end{gathered}
$$

Proof. If in the interval $[\alpha, \beta]$ there are no moments of impusle effect, i.e. $i[\alpha, \beta]=0$, then Theorem 1 is a consequence of the theorem of continuous dependence of the solutions of ordinary differential systems on initial data and a parameter $[3,4]$. $[\alpha, \beta]$.

Without loss of generality we assume that the points $\tau_{k}, k=1, \ldots, p$ lie in the interval

Since $\phi\left(\tau_{k}+0\right) \in \Omega, k=1, \ldots, p$, and the function $\phi(t)$ is continuous in $\left(\tau_{k-1}, \tau_{k}\right] \cap[\alpha, \beta]$, then the set

$$
\Gamma=\left\{(t, x) \in \mathbb{R}^{1+n}: \alpha \leqq t \leqq \beta, x=\phi(t)\right\} \cup \bigcup_{k=1}^{p}\left(\tau_{k}, \phi\left(\tau_{k}+0\right)\right)
$$

is a compact set contained in $I \times \Omega$. Then there exists a number $\delta^{*}>0$ such that the set

$$
Q=\left\{(t, x) \in \mathbb{R}^{1+n}: \alpha \leqq t \leqq \beta,|x-y| \leqq \delta^{*}, y \in \Gamma\right\}
$$


is contained in $I \times \Omega$. Let $\left.W=(t, x, \lambda) \in I \times \Omega \times \Lambda:(t, x) \in Q,\left|\lambda-\lambda^{*}\right| \leqq \delta^{*}\right\}$ and $M=$ $\sup _{W}|f(t, x, \lambda)|$. Since $W$ is a compact set, then the function $f$ satisfies the Lipschitz condition on $(x, \lambda)$ in $W$ globally, i.e. there exists a constant $L>0$ such that for $(t, x, \lambda),(t, y, \mu) \in W$ the following inequality holds

$$
|f(t, x, \lambda)-f(t, y, \mu)| \leqq L(x-y|+| \lambda-\mu \mid) .
$$

Let $x(t, \lambda)$ be a solution of the system of ordinary differential equations

$$
\frac{d x}{d t}=f(t, x, \lambda)
$$

which is defined for $t \in J \subset\left(\tau_{k-1}, t_{k}\right] \cap[\alpha, \beta], k=1,2, \ldots, p+1$.

Then for $t, \tau \in J$ we have

$$
\begin{gathered}
\phi(t)=\phi(\tau)+\int_{\tau}^{t} f\left(s, \phi(s), \lambda^{*}\right) d s, \\
x(t, \lambda)=x(\tau, \lambda)+\int_{\tau}^{t} f(s, x(s, \lambda), \lambda) d s .
\end{gathered}
$$

If $|x(t, \lambda)-\phi(t)|<\delta^{*}$ and $\left|\lambda-\lambda^{*}\right|<\delta^{*}$, then (5) and (7) imply the inequality

$$
\begin{gathered}
|x(t, \lambda)-\phi(t)| \leqq|x(\tau, \lambda)-\phi(\tau)|+\int_{\tau}^{t} L\left(|x(s, \lambda)-\phi(s)|+\left|\lambda-\lambda^{*}\right|\right)|d s| \\
\leqq|x(\tau, \lambda)-\phi(\tau)|+L\left|\lambda-\lambda^{*}\right|(\beta-\alpha)+\int_{\tau}^{t} L|x(s, \lambda)-\phi(s)||d s| .
\end{gathered}
$$

We apply the generalized lemma of Gronwall-Bellman to (8) and obtain the estimate

$$
|x(t, \lambda)-\phi(t)| \leqq\left(|x(\tau, \lambda)-\phi(\tau)|+L(\beta-\alpha)\left|\lambda-\lambda^{*}\right|\right) e^{L(\beta-\alpha)} .
$$

Let $\delta_{0}=\delta^{*} / 2$. In view of estimate (9) and condition (iii) of Theorem 1 we conclude that we can successively choose the numbers $\delta_{i}\left(0<\delta_{i}<\delta_{i-1}\right), i=1, \ldots, p+1$, so that if

$$
\begin{gathered}
k=1, \ldots, p ; j=1, \ldots, p+1 ;\left|\lambda-\lambda^{*}\right|<\delta_{i} ; \tau \in\left(\tau_{j-1}, \tau_{j}\right] \cap[\alpha, \beta] ; \\
s \in\left(\tau_{j-1}, \tau_{j}\right] \cap[\alpha, \beta] ; t \in\left(\tau_{k-1}, \tau_{k+1}\right] \cap[\alpha, \beta] ; \\
|x-\phi(\tau)|<\delta_{i} ;\left|y-\phi\left(\tau_{k}\right)\right|<\delta_{i}\left|z-\phi\left(\tau_{k}+0\right)\right|<\delta_{i}
\end{gathered}
$$

then

$$
|x(s ; \tau, x, \lambda)-\phi(s)|<\delta_{i-1},
$$




$$
\begin{gathered}
\left|x\left(t ; \tau_{k}, y+I_{k}(y, \lambda), \lambda\right)-\phi(t)\right|<\delta_{i-1}, \\
\left|x\left(t ; \tau_{k}, z, \lambda\right)-\phi(t)\right|<\delta_{i-1} .
\end{gathered}
$$

We choose the number $\xi=\delta_{p+1}$ and the set $U$ according to (3).

1. Let $\left(t_{0}, x_{0}, \lambda_{0}\right) \in U$. Then there exists a unique solution $x(t)=x\left(t ; t_{0}, x_{0}, \lambda_{0}\right)$ of system (2) such that $x\left(t_{0}+0\right)=x_{0}$. Let this solution be defined for $t \in J$. In view of the choice of $\xi$ we conclude that for $t \in J \cap[\alpha, \beta]$ the estimate

$$
\left|x\left(t ; t_{0}, x_{0}, \lambda_{0}\right)-\phi(t)\right|<\frac{\delta^{*}}{2}<\delta^{*}
$$

holds.

Hence $x(t)$ is continuable in $[\alpha, \beta]$ and estimate $(10)$ holds for $t \in[\alpha, \beta]$.

Thus assertion 1 of Theorem 1 is proved.

2. Let $\varepsilon>0$ be given, $\left(t, t_{0}, x_{0}, \lambda_{0}\right) \in[\alpha, \beta] \times U,(s, \tau, z, \lambda) \in[\alpha, \beta] \times U, i[t, s]=i\left[t_{0}, \tau\right]=0$ and $x(t)=x\left(t ; t_{0}, x_{0}, \lambda_{0}\right)$.

Let $t_{0}<t, i\left[t_{0}, t\right]=1$ and $t_{0}<\tau_{k}<t$. From the theorem of continuous dependence of the solutions of the system of ordinary differential equations (6) it follows that there exists a number $\delta_{1}\left(0<\delta_{1}<\xi\right)$ such that if $\left|x-x\left(\tau_{k}+0\right)\right|<\delta_{1},\left|\lambda-\lambda_{0}\right|<\delta_{1}$ and $|s-t|<\delta_{1}$, then

$$
\left|x\left(s ; \tau_{k}, x, \lambda\right)-x\left(t ; \tau_{k}, x\left(\tau_{k}+0\right), \lambda_{0}\right)\right|<\varepsilon .
$$

From the continuity of the function $\psi_{k}(x, \lambda)$ it follows that there exists $\delta_{2}\left(0<\delta_{2}<\delta_{1}\right)$ such that if $\left|\lambda-\lambda_{0}\right|<\delta_{2},\left|y-x\left(\tau_{k}\right)\right|<\delta_{2}$, then

$$
\left|\psi_{k}(y, \lambda)-x\left(\tau_{k}+0\right)\right|=\left|\psi_{k}(y, \lambda)-\psi_{k}\left(x\left(\tau_{k}\right), \lambda_{0}\right)\right|<\delta_{1} .
$$

From the continuous dependence of the solutions of the system of ordinary differential equations (6) it follows that there exists $\delta\left(0<\delta<\delta_{2}\right)$ such that if $\left|\tau-t_{0}\right|<\delta$, $\left|z-x_{0}\right|<\delta,\left|\lambda-\lambda_{0}\right|<\delta$, then

$$
\left|x\left(\tau_{k} ; \tau, z, \lambda\right)-x\left(\tau_{k} ; t_{0}, x_{0}, \lambda_{0}\right)\right|<\delta_{2} .
$$
then

In view of the choice of $\delta$ we conclude that if $|s-t|<\delta,\left|\tau-t_{0}\right|<\delta,\left|z-x_{0}\right|<\delta,\left|\lambda-\lambda_{0}\right|<\delta$,

$$
\left|x(s ; \tau, z, \lambda)-x\left(t ; t_{0}, x_{0}, \lambda_{0}\right)\right|<\varepsilon
$$

which proves the continuity of the function $x(s ; \tau, z, \lambda)$ at the point $\left(t, t_{0}, x_{0}, \lambda_{0}\right)$.

If $i\left[t_{0}, t\right]=p>1$, then assertion 2 is proved by applying the above arguments several times.

In the case $t<t_{0}$ assertion 2 is proved similarly by using the continuity of the functions $\Psi_{k}^{-1}(x, \lambda), k=1, \ldots, p$.

Thus assertion 2 is proved. 
3. Let $\varepsilon>0$ be given, $\left(t_{0}, x_{0}, \lambda_{0}\right) \in U,\left(\tau, y_{0}, \mu_{0}\right) \in U, \tau_{k}<\tau<x<\min \left(\tau_{k+1}, \beta\right)$ and let $\chi$ be fixed.

Applying the lemma of Gronwall-Bellman, as when obtaining estimate (9), we obtain that for $t \in[\tau, \chi]$ the inequality

$$
\left|x\left(t ; \tau, y_{0}, \mu_{0}\right)-x\left(t ; \tau, x_{0}, \lambda_{0}\right)\right| \leqq K\left(\left|y_{0}-x_{0}\right|+\left|\mu_{0}-\lambda_{0}\right|\right)
$$

is fulfilled where $K=e^{L(\beta-\alpha)}(L(\beta-\alpha)+1)$.

We denote $\eta=x\left(\tau ; \tau_{k}, x_{0}, \lambda_{0}\right), w=x\left(x ; \tau, \eta, \lambda_{0}\right), u=x\left(x ; \tau, x_{0}, \lambda_{0}\right), v=x\left(x ; \tau, y_{0}, \mu_{0}\right)$.

From the equality

$$
\begin{aligned}
& x\left(s ; \tau, y_{0}, \mu_{0}\right)-x\left(t ; \tau_{k}, x_{0}, \lambda_{0}\right) \\
& =x\left(s ; \varkappa, v, \mu_{0}\right)-x\left(t ; \varkappa, u, \lambda_{0}\right) \\
& \quad+x\left(t ; \varkappa, u, \lambda_{0}\right)-x\left(t ; \varkappa, w, \lambda_{0}\right)
\end{aligned}
$$

and assertion 2 of Theorem 1 it follows that there exists $\delta_{1}\left(0<\delta_{1}<\varepsilon\right)$ such that if

$$
|s-t|<\delta_{1},|v-u|<\delta_{1},|w-u|<\delta_{1},\left|\mu_{0}-\lambda_{0}\right|<\delta_{1}
$$

then

$$
\left|x\left(s ; \tau, y_{0}, \mu_{0}\right)-x\left(t ; \tau_{k}, x_{0}, \lambda_{0}\right)\right|<\varepsilon
$$

But since

$$
\begin{aligned}
& |v-u|=\left|x\left(x ; \tau, y_{0}, \mu_{0}\right)-x\left(x ; \tau, x_{0}, \lambda_{0}\right)\right| \leqq K\left(\left|y_{0}-x_{0}\right|+\left|\mu_{0}-\lambda_{0}\right|\right), \\
& |w-u|=\left|x\left(x ; \tau, \eta, \lambda_{0}\right)-x\left(x ; \tau, x_{0}, \lambda_{0}\right)\right| \leqq K\left|\eta-x_{0}\right| \leqq K M\left|\tau-\tau_{k}\right|
\end{aligned}
$$

then there exists $\delta\left(0<\delta<\delta_{1}\right)$ such that if

$$
|s-t|<\delta, \tau_{k}<\tau<\tau_{k}+\delta,\left|y_{0}-x_{0}\right|<\delta,\left|\mu_{0}-\lambda_{0}\right|<\delta
$$

then inequalities (11) are fulfilled, hence (12) as well.

Thus the limit relation (4.1) is proved. Relations (4.2)-(4.8) are proved similarly.

Thus Theorem 1 is proved.

For the linear system with impulse effect

$$
\begin{gathered}
\frac{d x}{d t}=A(t, \lambda) x, \quad t \neq \tau_{k}, \\
\left.\Delta x\right|_{t=\tau_{k}}=B_{k}(\lambda) x, \quad k=1,2, \ldots,
\end{gathered}
$$


where $A(t, \lambda)$ and $B_{k}(\lambda)$ are $(n \times n)$-matrices we formulate the following theorem as a corollary of Theorem 1 .

Theorem 2. Let the following conditions be fulfilled:

(i) The matrix-valued function $A(t, \lambda)$ is continuous in every set $\left(\tau_{k-1}, \tau_{k}\right] \times \Lambda, k=1,2, \ldots$ and for every $k=1,2, \ldots$ and $\lambda_{0} \in \Lambda$ there exists a finite limit

$$
\lim _{\substack{(t, \lambda) \rightarrow\left(\tau_{k}, \lambda_{0}\right) \\ i>\tau_{k}}} A(t, \lambda)
$$

(ii) The matrix-valued functions $B_{k}(\lambda)$ are continuous in $\Lambda$ and

$$
\operatorname{det}\left(E_{n}+B_{k}(\lambda)\right) \neq 0, \quad k=1,2, \ldots, \lambda \in \Lambda .
$$

Then the solution $x\left(t ; t_{0}, x_{0}, \lambda_{0}\right)$ of system (13) is a continuous function for $t \in I, t_{0} \in I$, $t \neq \tau_{k}, t_{0} \neq \tau_{k}, k=1,2, \ldots, x_{0} \in \mathbb{R}^{n}, \lambda_{0} \in \Lambda$ and the relations (4) hold with $I_{k}(x, \lambda)=B_{k}(\lambda) x$.

\subsection{Differentiability of the solutions}

Theorem 3. Let the following conditions be fulfilled:

(i) The function $f: I \times \Omega \times \Lambda \rightarrow \mathbb{R}^{n}$ is continuous in $\left(\tau_{k-1}, \tau_{k}\right] \times \Omega \times \Lambda, k=1,2, \ldots$, and the functions $\partial f / \partial x$ and $\partial f / \partial \lambda$ are continuous in $\left(\tau_{k-1}, \tau_{k}\right) \times \Omega \times \Lambda, k=1,2, \ldots$.

(ii) For every $\left(x_{0}, \lambda_{0}\right) \in \Omega \times \Lambda, k=1,2, \ldots$ there exist finite limits of the functions $f, \partial f / \partial x$ and $\partial f / \partial \lambda$ as $(t, x, \lambda) \rightarrow\left(\tau_{k}, x_{0}, \lambda_{0}\right), t<\tau_{k}$ and as $(t, x, \lambda) \rightarrow\left(\tau_{k}, x_{0}, \lambda_{0}\right), t>\tau_{k}$.

(iii) For $k=1,2, \ldots$ the mapping

$$
\psi_{k}: \Omega \times \Lambda \rightarrow \Omega \times \Lambda,(x, \lambda) \rightarrow(z, \lambda), z=\psi_{k}(x, \lambda)=x+I_{k}(x, \lambda)
$$

is a diffeomorphism.

(iv) For $\lambda=\lambda_{0} \in \Lambda$ system (2) has a solution $\psi(t)$ defined for $t \in[\alpha, \beta] ; \alpha, \beta \neq \tau_{k}, k=$ $1,2, \ldots$.

Then:

1. There exists $\delta>0$ such that the solution $\phi(t)=x\left(t ; t_{0}, x_{0}, \lambda_{0}\right)$ of system (2) has continuous partial derivatives $\partial x / \partial t, \partial x / \partial t_{0}, \partial x / \partial x_{0}, \partial x / \partial \lambda$ in the domain

$$
V: \alpha<t<\beta ; \alpha<t_{0}<\beta ; t, t_{0} \neq \tau_{k}, k=1,2, \ldots ;\left|x_{0}-\psi\left(t_{0}+0\right)\right|<\delta ;\left|\lambda-\lambda_{0}\right|<\delta .
$$

2. The partial derivative $u=\left(\partial x / \partial x_{0}\right)\left(t ; t_{0}, x_{0}, \lambda\right)$ is a solution of the initial value problem

$$
\begin{gathered}
\frac{d u}{d t}=\frac{\partial f}{\partial x}(t, \phi(t), \lambda) u, \quad t \neq \tau_{k}, \\
\left.\Delta u\right|_{t=\tau_{k}}=\frac{\partial I_{k}}{\partial x}\left(\phi\left(\tau_{k}\right), \lambda\right) u, \quad k=1,2, \ldots, \\
u\left(t_{0}+0\right)=E_{n} .
\end{gathered}
$$


3. The partial derivative $v=(\partial x / \partial \lambda)\left(t ; t_{0}, x_{0}, \lambda\right)$ is a solution of the initial value problem

$$
\begin{gathered}
\frac{d v}{d t}=\frac{\partial f}{\partial x}(t, \phi(t), \lambda) v+\frac{\partial f}{\partial \lambda}(t, \phi(t), \lambda), \quad t \neq \tau_{k}, \\
\left.\Delta v\right|_{t=\tau_{k}}=\frac{\partial I_{k}}{\partial x}\left(\phi\left(\tau_{k}\right), \lambda\right) v+\frac{\partial I_{k}}{\partial \lambda}\left(\phi\left(\tau_{k}\right), \lambda\right), \quad k=1,2, \ldots, \\
v\left(t_{0}+0\right)=0_{n m} .
\end{gathered}
$$

4. The partial derivative $\partial x / \partial t_{0}$ satisfies the relation

$$
\frac{\partial x}{\partial t_{0}}\left(t ; t_{0}, x_{0}, \lambda\right)=-\frac{\partial x}{\partial x_{0}}\left(t ; t_{0}, x_{0}, \lambda\right) f\left(t_{0}, x_{0}, \lambda\right)
$$

Proof. First we shall prove the existence of the partial derivative of the solution $x\left(t ; t_{0}, x_{0}, \lambda\right)$ with respect to the $i$ th coordinate of $x_{0}$. By virtue of Theorem 1 there exists $\delta>0$ such that the function $x\left(t ; t_{0}, x_{0}, \lambda\right)$ is defined and continuous in the set $V$ defined by relations (14).

Let $|h|<r$ where $r>0$ is small enough and $t_{0}, x_{0}, \lambda$ are fixed and satisfy relations (14).

We denote

$$
\begin{gathered}
x(t, h)=x\left(t ; t_{0}, x_{0}+h e_{i}, \lambda\right), \\
\Delta x(t, h)=x(t, h)-x(t, 0), \\
z(t, h)=\frac{1}{h} \Delta x(t, h),
\end{gathered}
$$

where $e_{i}=(0, \ldots, 1, \ldots, 0)$ is the $i$ th basis vector.

From Theorem 1 it follows that

$$
\lim _{h \rightarrow 0} x(t, h)=x(t, 0)=x\left(t ; t_{0}, x_{0}, \lambda\right)
$$

uniformly on $t \in[\alpha, \beta], t \neq \tau_{k}, k=1,2, \ldots$.

Applying the formula of Newton-Leibniz we obtain that for $h \neq 0$

$$
\begin{aligned}
\frac{d z}{d t}(t, h) & =\frac{1}{h}(f(t, x(t, h), \lambda)-f(t, x(t, 0), \lambda)) \\
& =\int_{0}^{1} \frac{\partial f}{\partial x}(t, x(t, 0)+s \Delta x(t, h), \lambda) d s z(t, h), \\
z\left(\tau_{k}+0, h\right)= & \frac{1}{h}\left(x\left(\tau_{k}, h\right)-x\left(\tau_{k}, 0\right)\right)+\frac{1}{h}\left(I_{k}\left(x\left(\tau_{k}, h\right), \lambda\right)-I_{k}\left(x\left(\tau_{k}, 0, \lambda\right)\right)\right.
\end{aligned}
$$




$$
\begin{aligned}
& =z\left(\tau_{k}, h\right)+\int_{0}^{1} \frac{\partial I_{k}}{\partial x}\left(x\left(\tau_{k}, 0\right)+s \Delta x\left(\tau_{k}, h\right), \lambda\right) d s z\left(\tau_{k}, h\right), \\
& z\left(t_{0}+0, h\right)=\frac{1}{h}\left(x\left(t_{0}+0, h\right)-x\left(t_{0}+0,0\right)\right)=e_{i}
\end{aligned}
$$

i.e. $z(t, h)$ is a solution of the initial value problem

$$
\begin{gathered}
\frac{d z}{d t}=A(t, h) z, \quad t \neq \tau_{k}, \\
\left.\Delta z\right|_{t=t_{k}}=B_{k}(h) z, \quad k=1,2, \ldots, \\
z\left(t_{0}+0\right)=e_{i},
\end{gathered}
$$

where

$$
\begin{aligned}
& A(t, h)=\int_{0}^{1} \frac{\partial f}{\partial x}(t, x(t, 0)+s \Delta x(t, h), \lambda) d s, \\
& B_{k}(h)=\int_{0}^{1} \frac{\partial I_{k}}{\partial x}\left(x\left(\tau_{k}, 0\right)+s \Delta x\left(\tau_{k}, h\right), \lambda\right) d s .
\end{aligned}
$$

The functions $A(t, h)$ and $B_{k}(h)$ satisfy the conditions of Theorem 2. Therefore,

$$
\lim _{h \rightarrow 0} z(t, h)=y(t)
$$

where $y(t)$ is the solution of the initial value problem

$$
\begin{gathered}
\frac{d y}{d t}=\frac{\partial f}{\partial x}\left(t, x\left(t ; t_{0}, x_{0}, \lambda\right), \lambda\right) y, \quad t \neq \tau_{k}, \\
\left.\Delta y\right|_{t=\tau_{k}}=\frac{\partial I_{k}}{\partial x}\left(x\left(\tau_{k} ; t_{0}, x_{0}, \lambda\right), \lambda\right) y, \quad k=1,2, \ldots, \\
y\left(t_{0}+0\right)=e_{i},
\end{gathered}
$$

which is obtained from (18) by setting $h=0$.

Thus we have proved the existence of the partial derivative of the solution $x\left(t ; t_{0}, x_{0}, \lambda\right)$ with respect to the $i$ th coordinate of $x_{0}$ and along with it the existence of $\left(\partial x / \partial x_{0}\right)\left(t ; t_{0}, x_{0}, \lambda\right)$. In view of (19) we conclude that the matrix $u=\left(\partial x / \partial x_{0}\right)$ is a solution of the initial value problem (15) and applying Theorem 2 we conclude that the solution $\left(\partial x / \partial x_{0}\right)\left(t ; t_{0}, x_{0}, \lambda\right)$ of the initial value problem (15) is a continuous function for $\left(t, t_{0}, x_{0}, \lambda\right) \in V$. 
The existence and continuity of $\partial x / \partial t_{0}, \partial x / \partial \lambda$ is proved in a similar way using the scheme of arguments used in the proof of analogous results for systems of ordinary differential equations $[3,4]$. Thus Theorem 3 is proved.

Equations (15) and (16) are analogues to the variational equations for a system of the form (2).

In the theory of systems with impulse effect, beside system (2), systems of the following forms are considered as well:

$$
\begin{gathered}
\frac{d x}{d t}=f(t, x, \lambda), \quad t \neq \tau_{k}(x), \\
\left.\Delta x\right|_{t=\tau_{k}(x)}=I_{k}(x, \lambda)
\end{gathered}
$$

and

$$
\begin{gathered}
\frac{d x}{d t}=f(x, \lambda), \quad x \bar{\epsilon} M, \\
\left.\Delta x\right|_{x \in M}=I(x, \lambda) .
\end{gathered}
$$

For system (20) it is characteristic that the impulse effect occurs in the moment $t$ when the mapping point $(t, x(t))$ meets some of the hypersurfaces $\sigma_{k} \equiv t=\tau_{k}(x)$, i.e. when the equality $t=\tau_{k}(x(t))$ is fulfilled.

For system (21) the impulse effect occurs when the point $x(t)$ hits the subset $M$ of the phase space. We shall note that system (21) has the property of autonomy.

The investigation of systems (20) and (21) is made difficult by the following peculiarities:

(a) The moments of impulse effect of the different solutions are different.

(b) For systems (20) and (21) the so called "beating" of the solutions is possible, i.e. the phenomenon when the mapping point $(t, x(t))$ (or $x(t)$ for system (21)) meets successively one and the same hypersurface $\sigma_{k}$ (or the set $M$ ) several or infinitely many times.

We shall note that in $[5,13]$ sufficient conditions for the absence of "beating" are given.

We shall give the variational equations for systems (20) and (21).

Let $\phi(t)=x\left(t ; t_{0}, x_{0}, \lambda\right)$ be a solution of system (20) with moments of impulse effect $t_{k}$ and $\phi_{k}=\phi\left(t_{k}\right), \phi_{k}^{+}=\phi\left(t_{k}+0\right), u=\left(\partial x / \partial x_{0}\right)\left(t ; t_{0}, x_{0}, \lambda\right), v=(\partial x / \partial \lambda)\left(t ; t_{0}, x_{0}, \lambda\right)$. Then

$$
\begin{gathered}
\frac{d u}{d t}=\frac{\partial f}{\partial x}(t, \phi(t), \lambda) u, \quad t \neq t_{k}, \\
\left.\Delta u\right|_{t=t_{k}}=\frac{\partial I_{k}}{\partial x}\left(\phi_{k}, \lambda\right) u+P_{k} \frac{\left(\partial \tau_{k} / \partial x\right)\left(\phi_{k}\right) u}{1-\left(\partial \tau_{k} / \partial x\right)\left(\phi_{k}\right) f\left(t_{k}, \phi_{k}, \lambda\right)}, \\
u\left(t_{0}+0\right)=E_{n} ;
\end{gathered}
$$




$$
\begin{gathered}
\frac{d v}{d t}=\frac{\partial f}{\partial x}(t, \phi(t), \lambda) v+\frac{\partial f}{\partial \lambda}(t, \phi(t), \lambda), \quad t \neq t_{k}, \\
\left.\Delta v\right|_{t=t_{k}}=\frac{\partial I_{k}}{\partial x}\left(\phi_{k}, \lambda\right) v+\frac{\partial I_{k}}{\partial \lambda}\left(\phi_{k}, \lambda\right)+P_{k} \frac{\left(\partial \tau_{k} / \partial x\right)\left(\phi_{k}\right) v}{1-\left(\partial \tau_{k} / \partial x\right)\left(\phi_{k}\right) f\left(t_{k}, \phi_{k}, \lambda\right)}, \\
v\left(t_{0}+0\right)=0_{n m},
\end{gathered}
$$

where

$$
P_{k}=\frac{\partial I_{k}}{\partial x}\left(\phi_{k}, \lambda\right) f\left(t_{k}, \phi_{k}, \lambda\right)+f\left(t_{k}, \phi_{k}, \lambda\right)-f\left(t_{k}, \phi_{k}^{+}, \lambda\right)
$$

Let $\phi(t)=x\left(t ; x_{0}, \lambda\right)$ be a solution of the autonomous system (21) with moments of impulse effect $t_{k}$ and $\phi_{k}=\phi\left(t_{k}\right), \phi_{k}^{+}=\phi\left(t_{k}+0\right)$ and $u=\left(\partial x / \partial x_{0}\right)\left(t ; x_{0}, \lambda\right), v=(\partial x / \partial \lambda)\left(t ; x_{0}, \lambda\right)$.

Let the set $M$ be given by the equation $\Phi(x)=0$ where $\Phi: \mathbb{R}^{n} \rightarrow \mathbb{R}$.

Then

$$
\begin{gathered}
\frac{d u}{d t}=\frac{\partial f}{\partial x}(\phi(t), \lambda) u, \quad t \neq t_{k}, \\
\left.\Delta u\right|_{t=t_{k}}=\frac{\partial I}{\partial x}\left(\phi_{k}, \lambda\right) u+Q_{k} \frac{(\partial \Phi / \partial x)\left(\phi_{k}\right) u}{(\partial \Phi / \partial x)\left(\phi_{k}\right) f\left(\phi_{k}, \lambda\right)}, \\
u\left(0_{+}\right)=E_{n} ; \\
\frac{d v}{d t}=\frac{\partial f}{\partial x}(\phi(t), \lambda) v+\frac{\partial f}{\partial \lambda}(\phi(t), \lambda), \quad t \neq t_{k}, \\
\left.\Delta v\right|_{t=t_{k}}=\frac{\partial I}{\partial x}\left(\phi_{k}, \lambda\right) v+\frac{\partial I}{\partial \lambda}\left(\phi_{k}, \lambda\right)+Q_{k} \frac{(\partial \Phi / \partial x)\left(\phi_{k}\right) v}{(\partial \Phi / \partial x)\left(\phi_{k}\right) f\left(\phi_{k}, \lambda\right)}, \\
v\left(0_{+}\right)=0_{n m}, \quad
\end{gathered}
$$

where

$$
Q_{k}=f\left(\phi_{k}^{+}, \lambda\right)-f\left(\phi_{k}, \lambda\right)-\frac{\partial I}{\partial x}\left(\phi_{k}, \lambda\right) f\left(\phi_{k}, \lambda\right)
$$

The variational equations for systems (20) and (21) are obtained by arguments similar to those in the proof of Theorem 3. Conditions excluding the phemomenon "beating" as well as the following conditions are added: 


$$
\frac{\partial \tau_{k}}{\partial x}\left(\phi_{k}\right) f\left(t_{k}, \phi_{k}, \lambda\right) \neq 1 \quad \text { (for system (20)) }
$$

and

$$
\frac{\partial \Phi}{\partial x}\left(\phi_{k}\right) f\left(\phi_{k}, \lambda\right) \neq 0 \quad(\text { for system }(21))
$$

Remark 1. Condition (26) means that the integral curve $(t, \phi(t))$ of system (20) is not tangent to the hypersurface $\sigma_{k}$ in the moment $t_{k}$. The interpretation of condition (27) is similar.

Remark 2. In the case when $\tau_{k}(x) \equiv \tau_{k}$ we have $\left(\partial \tau_{k} / \partial x\right) \equiv 0$ and the equations (22) and (23) coincide with the equations (15) and (16) respectively.

Remark 3. Let $\xi(t)$ be a solution of system (21) with moments of impulse effect $t_{k}$ and $\xi_{k}=\xi\left(t_{k}\right), \xi_{k}^{+}=\xi\left(t_{k}+0\right)$.

Then $\eta=(d \xi / d t)$ is a solution of the initial value problem

$$
\begin{gathered}
\frac{d u}{d t}=\frac{\partial f}{\partial x}(\xi(t), \lambda) u, \quad t \neq t_{k}, \\
\left.\Delta u\right|_{t=t_{k}}=\frac{\partial I}{\partial x}\left(\xi_{k}, \lambda\right) u+Q_{k} \frac{(\partial \Phi / \partial x)\left(\xi_{k}\right) u}{(\partial \Phi / \partial x)\left(\xi_{k}\right) f\left(\xi_{k}, \lambda\right)}, \\
u\left(0_{+}\right)=\frac{d \xi}{d t}\left(0_{+}\right),
\end{gathered}
$$

where

$$
Q_{k}=f\left(\xi_{k}^{+}, \lambda\right)-f\left(\xi_{k}, \lambda\right)-\frac{\partial I}{\partial x}\left(\xi_{k}, \lambda\right) f\left(\xi_{k}, \lambda\right)
$$

Indeed, for $t \neq t_{k}$ we have

$$
\frac{d \eta}{d t}=\frac{d^{2} \xi}{d t^{2}}=\frac{d}{d t} f(\xi(t), \lambda)=\frac{\partial f}{\partial x}(\xi(t), \lambda) \frac{d \xi}{d t}=\frac{\partial f}{\partial x}(\xi(t), \lambda) \eta
$$

and for $t=t_{k}$ we have

$$
\left.\Delta \eta\right|_{t=t_{k}}=\frac{d \xi}{d t}\left(t_{k}+0\right)-\frac{d \xi}{d t}\left(t_{k}\right)=f\left(\xi_{k}^{+}, \lambda\right)-f\left(\xi_{k}, \lambda\right)
$$

From (29) and (28.3) it follows that $\eta(t)=u(t)$ for $t \in\left[0, t_{1}\right]$ and, in particular, $u\left(t_{1}\right)=$ $\eta\left(t_{1}\right)=(d \xi / d t)\left(t_{1}\right)=f\left(\xi_{1}, \lambda\right)$.

Then by $(28.2)$ we have that $\Delta u\left(t_{1}\right)=f\left(\xi_{1}^{+}, \lambda\right)-f\left(\xi_{1}, \lambda\right)$, i.e. $u_{1}^{+}=\eta_{1}^{+}$. Further on the identity $u(t)=\eta(t)$ for $t \in\left(t_{k-1}, t_{k}\right], k=1,2, \ldots$, is proved by induction on $k$. 
3.3. Formula of variation of parameters for systems with impulse effect

Consider the systems

$$
\begin{gathered}
\frac{d x}{d t}=A(t) x, \quad t \neq \tau_{k}, \\
\left.\Delta x\right|_{t=\tau_{k}}=B_{k} x, \quad k=1,2, \ldots
\end{gathered}
$$

and

$$
\begin{gathered}
\frac{d x}{d t}=A(t) x+g(t, x), \quad t \neq \tau_{k}, \\
\left.\Delta x\right|_{t=\tau_{k}}=B_{k} x+h_{k}(x), \quad k=1,2, \ldots,
\end{gathered}
$$

where the $(n \times n)$-matrices $A(t)$ and $B_{k}$ and the functions $g: I \times \Omega \rightarrow \mathbb{R}^{n}$ and $h_{k}: \Omega \rightarrow \mathbb{R}^{n}$, $k=1,2, \ldots$ satisfy the following conditions which provide the existence, uniqueness and continuability of the solutions of systems (31) and (32):

1. The matrix $A(t)$ is continuous for $t \in I, t \neq \tau_{k}$, at the points $\tau_{k}(k=1,2, \ldots)$ it has discontinuities of the first type and is left continuous.

2. The function $g: I \times \Omega \rightarrow \mathbb{R}^{n}$ is continuous in $\left(\tau_{k-1}, \tau_{k}\right] \times \Omega, k=1,2, \ldots$.

3. The function $g$ is locally Lipschitz continuous on $x$ in $I \times \Omega$.

4. For any $x_{0} \in \Omega$ and $k=1,2, \ldots$ there exists a finite limit of $g(t, x)$ as $(t, x) \rightarrow\left(\tau_{k}, x_{0}\right)$, $t>\tau_{k}$.

Let $U_{k}(t, s)\left(t, s \in\left(\tau_{k-1}, \tau_{k}\right]\right)$ be the Cauchy matrix for the linear system

$$
\frac{d x}{d t}=A(t) x, \quad\left(\tau_{k-1}<t \leqq \tau_{k}\right)
$$

and $W(t, s)$ be the Cauchy matrix for system (31):

$$
W(t, s)=\left\{\begin{array}{l}
U_{k}(t, s) \quad \text { for } t, s \in\left(\tau_{k-1}, \tau_{k}\right], \\
U_{k+1}\left(t, \tau_{k}+0\right)\left(E_{n}+B_{k}\right) U_{k}\left(\tau_{k}, s\right) \text { for } \tau_{k-1}<s \leqq \tau_{k}<t \leqq \tau_{k+1}, \\
U_{k}\left(t, \tau_{k}\right)\left(E_{n}+B_{k}\right)^{-1} U_{k+1}\left(\tau_{k}+0, s\right) \text { for } \tau_{k-1}<t \leqq \tau_{k}<s \leqq \tau_{k+1}, \\
U_{k+1}\left(t, \tau_{k}+0\right) \prod_{j=k}^{i+1}\left(E_{n}+B_{j}\right) U_{j}\left(\tau_{j}, \tau_{j-1}+0\right)\left(E_{n}+B_{i}\right) U_{i}\left(\tau_{i}, s\right) \\
\text { for } \tau_{i-1}<s \leqq \tau_{i}<\tau_{k}<t \leqq \tau_{k+1}, \\
U_{i}\left(t, \tau_{i}\right) \prod_{j=i}^{k-1}\left(E_{n}+B_{j}\right)^{-1} U_{j+1}\left(\tau_{j}+0, \tau_{j+1}\right)\left(E_{n}+B_{k}\right)^{-1} U_{k+1}\left(\tau_{k}+0, s\right) \\
\text { for } \tau_{i-1}<t \leqq \tau_{i}<\tau_{k}<s \leqq \tau_{k+1} .
\end{array}\right.
$$


By straightforward verification we ascertain that for the solution $x(t)=x\left(t ; t_{0}, x_{0}\right)$ of system (32) the following representation is valid

$$
x(t)=\left\{\begin{array}{r}
W\left(t, t_{0}+0\right) x_{0}+\int_{t_{0}}^{t} W(t, s) g(s, x(s)) d s+\sum_{t_{0}<\tau_{k}<t} W\left(t, \tau_{k}+0\right) h_{k}\left(x\left(\tau_{k}\right)\right) \\
\text { for } t \in J^{+}\left(t_{0}, x_{0}\right) \\
W\left(t, t_{0}+0\right) x_{0}+\int_{t_{0}}^{t} W(t, s) g(s, x(s)) d s-\sum_{t \leqq \tau_{k} \leqq t_{0}} W\left(t, \tau_{k}+0\right) h_{k}\left(x\left(\tau_{k}\right)\right) \\
\text { for } t \in J^{-}\left(t_{0}, x_{0}\right) .
\end{array}\right.
$$

Remark 4. If any of the conditions $\operatorname{det}\left(E_{n}+B_{k}\right) \neq 0$ where $k$ is such that $\tau_{k} \leqq t_{0}$, is not fulfilled, then only formula (33) holds.

3.4. Analogue of Alekseev's formula for systems with impulse effect

Let us consider the systems with impulse effect

$$
\begin{gathered}
\frac{d x}{d t}=f(t, x), \quad t \neq \tau_{k}, \\
\left.\Delta x\right|_{t=\tau_{k}}=I_{k}(x), \quad k=1,2, \ldots
\end{gathered}
$$

and

$$
\begin{gathered}
\frac{d y}{d t}=f(t, y)+g(t, y), \quad t \neq \tau_{k}, \\
\left.\Delta y\right|_{t=\tau_{k}}=I_{k}(y)+h_{k}(y), \quad k=1,2, \ldots .
\end{gathered}
$$

We assume that systems (35) and (36) satisfy the conditions of Theorem 3.

Let $x(t)=x\left(t ; t_{0}, x_{0}\right)$ and $y(t)=y\left(t ; t_{0}, x_{0}\right)$ be solutions of systems (35) and (36) respectively and $\Phi\left(t, t_{0}, x_{0}\right)=\left(\partial x / \partial x_{0}\right)\left(t ; t_{0}, x_{0}\right)$ be the fundamental matrix of the variational system

$$
\begin{gathered}
\frac{d u}{d t}=\frac{\partial f}{\partial x}\left(t, x\left(t ; t_{0}, x_{0}\right)\right) u, \quad t \neq \tau_{k}, \\
\left.\Delta u\right|_{t=\tau_{k}}=\frac{\partial I_{k}}{\partial x}\left(x\left(\tau_{k} ; t_{0}, x_{0}\right)\right) u, \quad k=1,2, \ldots, \\
u\left(t_{0}+0\right)=E_{n} .
\end{gathered}
$$


Consider the function $\phi(s)=x(t ; s, y(s))$. By virtue of the properties of $\Phi\left(t, t_{0}, x_{0}\right)$ we obtain

$$
\begin{gathered}
\frac{d \phi}{d s}=\frac{d x}{d s}(t ; s, y(s))=\Phi(t, s, y(s)) g(s, y(s)), \quad s \neq \tau_{k} ; \\
\left.\Delta \phi\right|_{s=\tau_{k}}=x\left(t ; \tau_{k}+0, y\left(\tau_{k}+0\right)\right)-x\left(t, \tau_{k}-0, y\left(\tau_{k}-0\right)\right) \\
=x\left(t ; \tau_{k}, y\left(\tau_{k}\right)+I_{k}\left(y\left(\tau_{k}\right)\right)+h_{k}\left(y\left(\tau_{k}\right)\right)\right)-x\left(t ; \tau_{k}, y\left(\tau_{k}\right)+I_{k}\left(y\left(\tau_{k}\right)\right)\right) \\
=\int_{0}^{1} \Phi\left(t, \tau_{k}, y\left(\tau_{k}\right)+I_{k}\left(y\left(\tau_{k}\right)\right)+\tau h_{k}\left(y\left(\tau_{k}\right)\right)\right) d \tau h_{k}\left(y\left(\tau_{k}\right)\right) ; \\
\\
\phi\left(t_{0}+0\right)=x\left(t ; t_{0}+0, y\left(t_{0}+0\right)\right)=x\left(t ; t_{0}, x_{0}\right) .
\end{gathered}
$$

Then for $t \neq \tau_{k}$ we have

$$
\phi(t)= \begin{cases}\phi\left(t_{0}+0\right)+\int_{t_{0}}^{t} \frac{d \phi}{d s} d s+\sum_{t_{0}<\tau_{k}<t} \Delta \phi\left(\tau_{k}\right) & \text { for } t>t_{0} \\ \phi\left(t_{0}+0\right)+\int_{t_{0}}^{t} \frac{d \phi}{d s} d s-\sum_{t \leqq \tau_{k} \leqq t_{0}} \Delta \phi\left(\tau_{k}\right) & \text { for } t \leqq t_{0}\end{cases}
$$

and since $\phi(t)=x(t ; t, y(t))=y(t)$, then

$$
y(t)= \begin{cases}x(t)+\int_{t_{0}}^{t} \Phi(t, s, y(s)) g(s, y(s)) d s \quad\left(t>t_{0}\right) \\ \quad+\sum_{t_{0}<\tau_{k}<t} \int_{0}^{1} \Phi\left(t, \tau_{k}, y\left(\tau_{k}\right)+I_{k}\left(y\left(\tau_{k}\right)\right)+\tau h_{k}\left(y\left(\tau_{k}\right)\right)\right) d \tau h_{k}\left(y\left(\tau_{k}\right)\right) \\ x(t)+\int_{t_{0}}^{t} \Phi(t, s, y(s)) g(s, y(s)) d s \quad\left(t \leqq t_{0}\right) \\ \quad-\sum_{t \leq \tau_{k} \leqq t_{0}} \int_{0}^{1} \Phi\left(t, \tau_{k}, y\left(\tau_{k}\right)+I_{k}\left(y\left(\tau_{k}\right)\right)+\tau h_{k}\left(y\left(\tau_{k}\right)\right)\right) d \tau h_{k}\left(y\left(\tau_{k}\right)\right)\end{cases}
$$

Formula (37) represents an analogue of Alekseev's formula $[1,6]$.

\section{REFERENCES}

1. V. M. Alekseev, An estimate for perturbations of solutions of ordinary differential equations, Vestnik Moskov. Univ. Ser. 1 Mat. Mekh. 2 (1961), 28-36.

2. D. D. Barnov and P. S. Simeonov, Integral and differential inequalities for a class of piecewise continuous functions, Czechoslovak Mat. J. 36 (111) (1986), 417-426 (in Russian). 
3. J. N. Bibixov, A General Course of Ordinary Differential Equations (Publ. House of Leningrad State University, 1981 (in Russian)).

4. E. A. Coddington and N. Levinson, Theory of Ordinary Differential Equations (McGraw-Hill, New York, Toronto, London, 1955).

5. A. B. Dishliev and D. D. Bainov, Conditions for the absence of the phenomenon "beating" for systems of impulse differential equations, Bull. Inst. Math. Acad. Sinica 13 (1985), 237-256.

6. M. E. LoRD and A. R. Mrtchell, A new approach to the method of nonlinear variation of parameters, J. Appl. Math. Comp., to appear.

7. V. D. Millman and A. D. Mrshisis, On the stability of motion in the presence of impulses, Siberian Math. J. 1 (1960), 233-237 (in Russian).

8. A. D. Myshkis and A. M. Samollenko, Systems with impulses at prescribed moments of the time, Matn. Sb. 74 (1967), 202-208 (in Russian).

9. S. G. Pandit and S. G. Deo, Differential Systems Involving Impulses (Springer-Verlag, 1982).

10. T. Pavlidis, Stability of systems described by differential equations containing impulses, IEEE Trans. 12 (1967), 43-45.

11. V. Raghavendra and M. Rama Mohana Rao, On stability of differential systems with respect to impulsive perturbations, J. Math. Anal Appl. 48 (1974), 515-526.

12. A. M. Samollenko, D. D. Bainov and S. D. Milusheva, On the application of the averaging method for systems of integro-differential equations of standard type with discontinuous righthand side, Acta Math. Acad. Sci. Hung. 29 (1977), 31-48.

13. A. M. Samollenko and N. A. Perestiuk, Stability of the solutions of differential equations with impulse effect, Differ. Uravn. 11(1977), 1981-1992 (in Russian).

14. A. M. Samollenko and N. A. Perestiuk, On the stability of systems with impulse effect, Differ. Uravn. 11 (1981), 1995-2001 (in Russian).

15. A. M. Samollenko, N. A. Perestiuk and M. U. Ahmetov, Almost periodic solutions of impulse differential equations, Kiev Inst. Math. Acad. Sci. of Ukr. SSR (1983) (Preprint 83.26, in Russian).

16. P. S. Simeonov and D. D. Barnov, Asymptotic equivalence of two systems of differential equations with impulse effect, Systems \& Control Lett. 3 (1983), 297-301.

17. P. S. Simeonov and D. D. Bainov, Stability under persistent disturbances for systems with impulse effect, J. Math. Anal. Appl. 109 (1985), 546-563.

18. P. S. Simeonov and D. D. Bainov, The second method of Liapunov for systems with an impulse effect, Tamkang J. Math. 16 (1985), 19-40.

19. P. S. Simeonov and D. D. Bainov, Stability with respect to part of the variables in systems with impulse effect, J. Math. Anal. Appl. 117 (1986), 247-263.

20. V. SReE Hari Rao, On boundedness of impulsively perturbed systems, Bull. Austral. Math. Soc. 18 (1978), 237-242.

Plovdiv University

"Paissi Hilendarski"

Plovin

Bulgaria 